\title{
Cochlear implantation in pontine tegmental cap dysplasia
}

\begin{abstract}
Pontine tegmental cap dysplasia (PTCD) is a rare disease associated with hearing loss. We present a case of a child with PTCD and Perventricular nodular heterotopia (PNH). PTCD is a brainstem, cerebellar and cranial nerve malformation syndrome characterized by several clinical manifestations. PNH is a condition in which neurons do not migrate properly in the first stage of brain development. Diagnosis of both pathologies is based on the characteristic neuro-image findings. The child was evaluated, in our department, for congenital profound hearing loss, several signs and symptoms emerged during the diagnostic analysis. The clinical data and Neuro-image tests evidenced a diagnosis of PTCD and PHN. Cochlear Implant (CI) therapy for hearing loss in this case is discussed.
\end{abstract}

CI treatment in cases with profound hearing loss in PTCD is useful. These patients need a challenging and complex rehabilitative program. An adequate evaluation of clinical and functional responses and a cost-benefit analysis is necessary in the choice of monolateral or bilateral CI. A multidisciplinary and multicenter consensus could be useful in the therapeutic management of similar cases.

Keywords: cochlear implant, pontine tegmental cap dysplasia, congenital hearing loss, auditory neuropathy
Volume 6 Issue 2 - 2017

\section{Eliana Cristofari,Annalisa Meli}

Maternal infant department Audio Vestibology Service Circolo e

Fondazione Macchi Hospital Asst settalaghi, Italy

Correspondence: Eliana Cristofari Maternal infant department Audio Vestibology ServiceCircolo e Fondazione Macchi Hospital Asst settalaghiViale Borri 56 21 I00 Varese, Italy, Email elinana.critofari@ass-settelaghi.it

Received: November 04, 2016 | Published: February 21, 2017
Abbreviations: PTCD, pontine tegmental cap dysplasia; PNH, perventricular nodular heterotopia; CI, cochlear implant; OS, ostium secundum; OAEs, oto acoustic emission; ABR, auditory brainstem response; SVR, cortical event-related potentials to auditory stimuli; CPA, cerebello pontine angle; IAC, internal auditory canal; AutoNRT, response telemetry; CT scan, computed tomography; RMI, magnetic resonance imaging; cVEMP, cervical-vestibular evoked myogenic potentials

\section{Introduction}

At the time of this writing, we are not aware of any documented cases of a combination of Pontine tegmental cap dysplasia (PTCD) and Perventricular nodular heterotopia (PNH), or an association of PNH with other brainstem malformation in whom profound hearing loss was treated with cochlear implant (CI). Diagnosis of both pathologies is based on the characteristic neuro-image findings.

PTCD is a brainstem, cerebellar and cranial nerve malformation syndrome characterized by several clinical manifestations. ${ }^{1}$ Reports report PTCD presents with cranial nerves involvement, facial nerve palsy, sensorineural hearing loss, corneal reflex alteration, mild to moderate cognitive impairment, swallowing and feeding disorders, ataxia, language impairment, deficit in visual-spatial tasks. ${ }^{1-3}$ Its pathogenesis and molecular mechanisms are unknown; a hypothesis considers an abnormal neuronal migration the cause of this malformation..$^{1-2}$ Other hypothesis is that the altered pontine anatomy is related to abnormal migration of pontine nuclei, where the cochlear ganglion cells may not establish normal synaptic connection with cochlear nuclei cells, and subsequently undergo apoptosis. PTCD is considered a syndrome of human genetic disorders of axon guidance, even if its etiology is unknown, but with no genetic cause identified to date. ${ }^{4}$

$\mathrm{PNH}$ is a condition in which neurons do not migrate properly in the first stage of brain development. The neurons that failed their migration form clumps around the ventricles. PNH is a type of malformation of cortical development. It can be sporadic or familiar; in some cases a genetic etiology is demonstrated, related to a chromosome 5 or $\mathrm{X}$-linked, caused by the mutation in ARFGEF2 and FLNA genes. Patients with PNH could be affected by mild intellectual disability and difficulty with spelling and reading, epilepsy of variable severity, and extracranial involvement especially cardiovascular deficits. PNH may be part of a more complex developmental disorder ${ }^{5}$ and generally is associated with mild hypoplasia of the corpus callosum and cerebellum. We report on our experiences with a case presenting with PTCD and PNH combined treated with two cochlear implants for hearing loss.

\section{Case presentation}

The patient was through caesarean section at 38 weeks of gestation. Pregnancy was normal; the mother reported taking only cardio aspirin, she is carrier of balanced translocation isht. ${ }^{4,6}$ A chorionic villus sampling was performed during pregnancy. Postnatal fetal karyotype was 46, XX, isht ${ }^{4,6}$ (p15.33, p13.3+; p13.3-,p15.33+). At birth, the baby's Apgar score was 8 at 1minute, 9 at 5minute and 10 at 10minute; her weight was $2540 \mathrm{~g}$, with a $32 \mathrm{~cm}$ head circumference. Icterus (jaundice), caused by ABO blood type incompatibility, was treated with phototherapy. The patient was not screened for hearing loss, for absence of neonatal hearing screening program in the hospital were the baby was born. A reduction of the age appropriate sucking reflex was also noted. After hospital discharge, the patient returned for diagnostic tests to check on the ensuing excessive weight loss. The following analyses results were normal: cerebral echography, plasmatic level of ammonium, levels of lactic acid and amino acid. The cardiac echography reveled an Ostium secundum (OS) type of atrial septum defect. A hearing loss was suspected by parents and during a second pediatric evaluation. At two months of age, the patient was sent for audiologic investigations in our center in November 2012. The external auditory canal and tympanic membrane were normal at otoscopic examination. 
An age appropriate audiologic test battery was performed which consisted of behavioral observation audiometry, otoacoustic emission (OAEs), Auditory Brainstem Response (ABR), threshold ABR, acoustic reflex threshold, tympanometry, and cortical event-related potentials to auditory stimuli (SVR). The test results revealed a bilateral, profound sensorineural hearing loss. No responses to OAEs and ABR were obtained; however, cortical event-related potentials were observed. Binaural hearing aids and a sternal vibrotactile device were prescribed and fitted. Aided thresholds for warble tones in the sound field were $80 \mathrm{~dB}$ HL and $60 \mathrm{~dB}$ HL with acoustic hearing aids and a vibrotactile device respectively. In view of the marginal benefit from the conventional and vibrotactile devices, further management of the child's hearing loss with a CI was suggested.

Prior to implant, the child underwent a rehabilitation program including tasks focusing on cognitive training and auditory training. The aim of the rehabilitation program was to improve listening skill prerequisites required for successful CI treatment. Following and during the rehabilitation training communication behavior analysis for children under age two years ${ }^{7}$ showed minor improvement in her responses relating to visual and auditory attention. In August 2013, the child was affected by corneal ulcer and hypoesthesia on the left eye. The ocular analysis evidenced an anterior segment malformation and a central leucoma.

The neurologic evaluation revealed psyco-motor and postural development delay, hypotonia, a facial and oro-pharyngeal hypoestesia, oral dyspraxia and swallowing disorders. The patient was evaluated with Griffiths Mental Developments Scales; ${ }^{8}$ the result showed a medium psychomotor development delay with an intelligence quotient of 57. Oculomotor disorders in the left eye, an extropia and leucocoria was confirmed. The child had an atrial septal defect with good functional compensation. Genetic analysis was normal for several possible mutations with known association related to hearing loss including: Connexin-26, Connexin-31, Connexin-43, mitocondrial A1555G, mitocondrial A7445G, microRNA 96, GJB6d13s1830 and GJB6-d13s1854 deletion mutation.

The temporal bones Computed Tomography (CT scan) and 1,5 Tesla Magnetic Resonance Imaging (RMI), performed after functional diagnosis, showed several anatomical malformations (Figure 1). The CT scan showed bilateral internal auditory canal stenosis; the axial and coronal measures of the canals were 1 millimeter for both sides. The MRI showed an absence of cochlear and vestibular nerve on the right side, and of the cochlear portion on the left side. Additional analyses were performed to further investigate the anatomical/ medical status prior to implant. Neuro-radiological imaging using a 3 Tesla MRI, to provide finer details of cerebral and neural structures, confirmed cochlear-vestibular nerve malformations, and evidenced finer detail of the anatomical deviations previously observed: brain stem malformation, flattening of the ventral pons and a median posterior "pontine cap". A horizontal pontine cleft was evidenced in the posterior pons. The bulbopontine junction was poorly defined, and a thinning of the ponsmesencephalic junction was observed. The median cerebellar peduncles appeared shorter and thinner than normal size.

The inner ear structures were normal on both sides. Hypoplasia of the internal auditory canals bilaterally was observed. Bilateral cochlea-vestibular nerves were not entirely recognizable. The facial nerve was visible on both sides, but the entry point was forward and anterior to the internal auditory canal. In normal condition the facial nerve and the nervus intermedius leave the brainstem near the dorsal pons at the pontomedullary junction (the cisternal segment of the facial nerve). Within the cerebellopontine angle (CPA), the nerve travels anterolaterally into the porus acousticus of the internal auditory canal (IAC), anterior to the vestibulocochlear nerve. ${ }^{9}$ In cases of severe malformations it's better to perform a stronger MRI to define anatomical details and to obtain indications useful for the treatment.

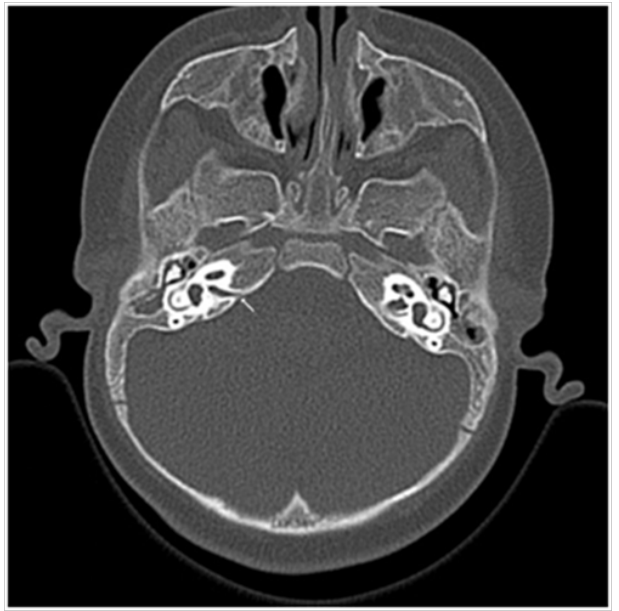

Figure I CT scans: normal inner ear and stenotic internal auditory canal.

A dysmorphism of the corpum callosum and subependimal noduli near the trigonus and in the posterior segment of the right temporal lobe was shown. The MRI results suggested a final diagnosis of pontine cap dysplasia (PTCD) and periventricular nodular heterotropia (PNH) (Figure 2\&3). To exclude that the behavioral responses depended from saccular hearing, further the cervical-vestibular evoked myogenic potentials (cVEMP) were measured to assess the presence of saccular and vestibular nerve responses were present but with low amplitude on the left side, while they were absent on the right side.

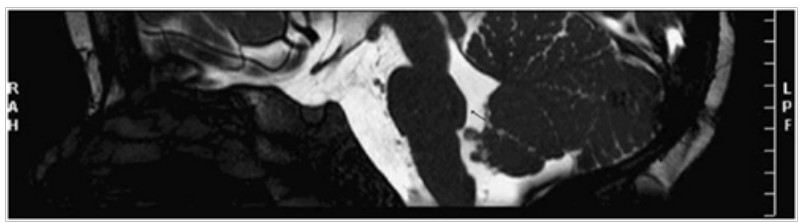

Figure 2 Saggital TI-weighted MRI image showing dysmorphism of the dorsal upper pons proteding in the fourth ventricle, flattening of the ventral pons.

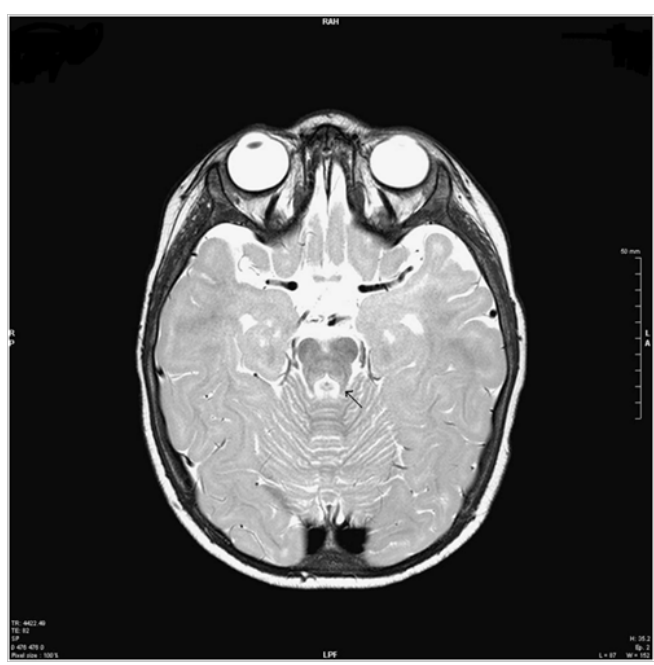

Figure 3 Axial T2-weighted MRI image showing molar-tooth-like sign. 
In January 2014, the child underwent unilateral CI surgery on the left side with a Cochlear Nucleus CI24RE Contour Advanced implant. The surgical approach was standard with no surgical events. The perimodular electrode array was introduced into the scala tympani through an anterio-inferior cochleostomy. Auto Neural Response Telemetry (Auto-NRT), responses were present for the basal electrodes (i.e. electrodes 2,3,4,5 and 6) with morphology lose to normal and with significantly elevated threshold. ${ }^{10}$ The postoperative course proceeded normally. Mean auditory thresholds measured with the CI alone, obtained through behavioral audiometry in the sound field for warble tones, was $50 \mathrm{~dB} \mathrm{HL}$ for the frequencies $(0.5,1.0,2.0$ and $4.0 \mathrm{kHz}$ ). The aided auditory thresholds for bilateral and bimodal stimulation with the $\mathrm{CI}$ in the left ear and a hearing aid in the right ear, resulting in a mean threshold for the same frequencies of $40 \mathrm{~dB} \mathrm{HL}$.

Following implant the child was enrolled into speech rehabilitation program, with three sessions per week at hospital and two sessions at home through video-conference for one year. With the acknowledgement that the child's communication behavior was well below the age appropriate norms preimplant, post implant the communication behavior analysis showed slow improvement in auditory attention over the first 12 months. In consideration of the improvement of auditory threshold gain and behavioral communication responses, despite morphological evidence in March 2015, the patient was operated for a sequential $2 \mathrm{nd} \mathrm{CI}$ in the right ear. As for the first surgery, the surgical approach was standard, with introduction of the contoured electrode array of a Cochlear Nucleus 512 implant into the scala tympani through the round window. Auditory thresholds in the bilateral CI condition for warble tones in the sound field were $50 \mathrm{~dB}$ HL. Auditory thresholds in the right CI condition for warble tones in the sound field was $60 \mathrm{~dB}$ HL. Auditory threshold in the left $\mathrm{CI}$ condition for warble tones in the sound field was $90 \mathrm{db}$ HL. Patient's communication skills improved after CI activation, but remained lower than age-related ability.

\section{Discussion}

Our patient showed neuro-radiological findings and clinical symptoms of PTCD: hypotonia, psyco-motor delay, feeding and swallowing disorders, bilateral sensorineural hearing loss, hypoesthesia and extropia in the left eye. Facial palsy was not present, despite the neuro-radiological analysis confirming an anomalous route of the VII nerve on both sides.

The patient displayed features of multiple cranial neuropathies. Feeding and swallowing disorders could be related to a glossopharyngeal nerve involvement or to the oro-pharyingeal hypoestesia. An oculomotor nerve damage could cause the exotropia the corneal hypoestesia is related to a trigeminal nerve involvement.

Audio logical findings evidenced cochlear dysfunction, as demonstrated by OAEs results, morphological analysis showing cochlear nerve disease. ABR indicated no response of the VIII nerve, but the presence of cortical event-related potentials to auditory stimuli evidenced the activation of the cortex to auditory stimulus. We speculated that auditory pathways function was partially preserved bilaterally. The first CI was performed on the left side, where functional and morphological data were better than right side. Patient's communicative performances improved with first CI Bimodal stimulation (CI+Hearing aid) showed a small binaural effect. Sequential right CI was supported by these data.

The pathophysiology of hearing loss is unknown, our patient showed inner ear damage and a VIII nerve pathology, with probably both mechanisms influencing the profound deafness. Similar findings have been reported for such cases by other authors. ${ }^{9,11}$

The sequential CI did not bring a binaural benefit in this patient. The Binaural Processor (BP) is localized some anatomical structures in the brainstem and in the cortex. In particular the Olivary Complex ${ }^{12}$ and the Collicolus. ${ }^{13}$ These structures process the auditory inputs from the crossed auditory pathways. It's possible that BP and auditory crossed pathways are altered by the brainstem malformations.

In our reported case study, in addition to the cochlear and auditory nerve pathologies observed, both facial nerves had an anomalous route; nevertheless, the electrical stimulation from either CI did not activate the facial nerves on either side.

In our child case report, CI was indicated for the cochlear pathology for the treatment of hearing loss, but the malformation observed of both VIII nerves is likely to lead to reduced outcomes and performance and subsequently expectations. We believe that the first CI was a good clinical choice of treatment in any case as it lead to the improved communicative performance after the activation of the left CI. The child is a full-time user of the first implant, and also of the sequential CI. Auditory attention, awareness improved after CI activation. Speech language therapist noted an improvement of patient collaboration during rehabilitative sessions. No negative side effects of bilateral CI were evidenced. Patient was also enrolled in a rehabilitative program for swallowing disorder and a psychomotor rehabilitation program. In agreement with the child psychiatrist and the speech therapist Augmentative and Alternative Communication strategies were suggested.

Bacciu reported successful CI results in a case of PTCD and suggested that the retrocochlear auditory function is at least partially intact in deaf patients with PTCD. ${ }^{11}$ CI results in their patient showed a good gain in auditory thresholds and an improvement of communication performances. CI treatment in cases with profound hearing loss in PTCD is useful. These patients need a challenging and complex rehabilitative program. An adequate evaluation of clinical and functional responses, and a cost-benefit analysis is necessary in the choice of monolateral or bilateral CI. A multidisciplinary and multicenter consensus could be useful in the therapeutic management of similar cases.

\section{Acknowledgments}

None.

\section{Conflicts of interest}

Author declares there are no conflicts of interest.

\section{Funding}

None.

\section{References}

1. Barth PG, Majoie CB, Caan MW, et al. Pontine tegmental cap dysplasia: a novel brain malformation with a defect in axonal guidance. Brain. 2007;130(Pt 9):2258-2266.

2. Jissendi-Tchofo P, Doherty D, McGillivray G, et al. Pontine tegmental cap dysplasia: MR imaging and diffusion tensor imaging features of impaired axonal navigation. AJNR Am J Neuroradiol. 2009;30(1):113-119.

3. Briguglio M, Pinelli L, Giordano L, et al. Pontine Tegmental Cap Dysplasia: developmental and cognitive outcome in three adolescent patients. Orphanet J Rare Dis. 2011;6:36. 
4. Engle EC. Human genetic disorders of axon guidance. Cold Spring Harb Perspect Biol. 2010;2(3):a001784.

5. Battaglia G1, Granata T. Perventricular nodular heterotopia. Handb Clin Neurol. 2008;87:177-189.

6. Gupta S, Mends F, Hagiwara M, et al. Imaging the facial nerve: a contemporary review. Radiology Research and Practice. 2013;2013:1-14.

7. Tait DM. Video Analysis: A method of assessing changes in pre-verbal and early linguistic communication following cochlear implantation. Ear Hear. 1993;14(6):378-379.

8. M Huntley. The Griffiths Mental Developmental Scales Manual from birth to two years. Bucks: Association for Research in Infant and Child Development, The Test Agency Thames, New Zealand. 1996. p. 5-39.
9. Maeoka Y, Yamamoto T, Ohtani K, et al. Pontine hypoplasia in a child with sensorineural deafness. Brain Dev. 1997;19(6):436-439.

10. Gordon KA, Papsin BC, Harrison RV. An evoked potential study of the developmental time course of the auditory nerve and brainstem in children using cochlear implants. Audiol Neurootol. 2006;11(1):7-23.

11. Bacciu A, Ormitti F, Pasanisi E, et al. Cochlear implantation in pontine tegmental cap dysplasia. Int $J$ Pediatr Otorhinolaryngol. 2010;74(8):962-966.

12. Caird D, Klinke R. Processing of binaural stimuli by cat superior olivary complex neurons. Experimental Brain Research. 1983;52(3):385-399.

13. Gockel HE, Carlyon RP, Plack CJ. Combination of spectral and binaurally created harmonics in a common central pitch processor. $J$ Assoc Res Otolaryngol. 2011;12(2):253-260. 